\title{
Análisis contrastivo de la jerga peruana de hace 10 años hasta la actualidad, 2019
}

\author{
SAGÁSTEGUI TORIBIO, Edwin Eduardo; RODRÍGUEZ RUIZ,Yosmiani Jacqueline; \\ MEDINA LESCANO, Miguel Ángel
}

\section{RESUMEN}

El siguiente proyecto se denomina "Análisis contrastivo de las jergas peruanas de hace 10 años hasta la actualidad, 2019". Este trabajo de corte descriptivo pretende hacer un análisis contrastivo de las jergas que aún se siguen utilizando en la actualidad con aquellas utilizadas en la década anterior. El objetivo principal de este trabajo de investigación fue identificar aquellas jergas que aún siguen en vigencia, así como las que ya no están vigentes al momento de realizar este trabajo. De la misma manera, este trabajo de investigación ofrece un listado de términos equivalentes de aquellas jergas en desuso. La muestra estuvo conformada por 60 estudiantes de 1er año del Programa de Traducción e Interpretación. Para tal fin, se utilizó un listado de jergas que ha sido producto de más de una investigación anterior, se las categorizó según la intencionalidad de esta investigación, es decir se usó aquellas que colindan con las situaciones cotidianas de estudiantes universitarios (mundo académico y no académico)

Posteriormente, se encuestaron a estudiantes universitarios sobre la vigencia de dichas jergas (aquellas que ya están en desuso y aquellas que siguen siendo utilizadas). Para las que ya están en desuso, se les pidió a los encuestados que coloquen un término equivalente. De esta manera pudimos verificar si la jerga ha sufrido alguna alteración en su uso contextual o no.

Se espera que la información obtenida como producto de nuestra investigación sirva de base para futuras investigaciones futuras que conlleven a un mejor entendimiento del funcionamiento de las jergas y sus cambios con el paso de los años.

Palabras clave: jergas, análisis contrastivo, equivalente

\section{Contrastive analysis of Peruvian slang from ten years ago to the present, 2019}

\begin{abstract}
The following project is called "Contrastive analysis of Peruvian slang from 10 years ago to the present, 2019". This descriptive study aims to make a contrastive analysis of the slang that is still used nowadays with that used in the previous decade. The main objective of this research was to identify those terms that are still used, as well as those that are no longer used at the time of this study. Likewise, this research work provides a list of equivalent terms of those terms in disuse. The sample was made up of 60 first-year students from the Translation and Interpretation Program. For this purpose, a list of terms that have been the product of more than one previous investigation was used. They were categorized according to the intent of this research, that is to say, those that occur in the daily situations of university students (academic and not academic world). Subsequently, university students were surveyed on the continuing validity of these terms (those that are already in disuse and those that continue to be used). For those already in disuse, respondents were asked to provide an equivalent term. In this way we were able to verify if the term had suffered any alteration in its contextual use or not. It is hoped that the information obtained as a result of our research will serve as a basis for future studies leading to a better understanding of how slang works and how it changes over the years.
\end{abstract}

Keywords: Slang, contrastive analysis, equivalent

Universidad César Vallejo. ORCID: 0000-0003-2230-9378, esagastegui@ucv.edu.pe

'Universidad César Vallejo. ORCID: 0000-0002-5836-4507, yosmianij@hotmail.com

3universidad César Vallejo. ORCID: 0000-0001-9813-6712, mmedinal@ucv.edu.pe 


\section{INTRODUCCIÓN}

El hablar cotidiano trae consigo un sinfín de situaciones que van desde lo profesional pasando por lo coloquial y llegando hasta el uso de las jergas. Las jergas, muchas veces desapercibidas, forman parte también de nuestro léxico habitual y como tal son susceptibles a cambios en su fondo y forma a través de los años. Existen diferentes tipos de jergas, por ejemplo, dentro de una profesión cualquiera puede identificar el léxico que se usa para hablar de situaciones netamente profesionales y correspondientes a un área específica. Asimismo, en el ámbito académico, también se usan diferentes jergas que son fáciles de entender para aquellas personas que están dentro de este círculo. No podemos negar tampoco la existencia de la jerga delincuencial que también forma parte del hablar, no solo de este grupo de personas, sino también de los medios de comunicación hablada y escrita. Como se puede ver, la jerga está presente en toda nuestra vida diaria y la usamos sin percatarnos muchas veces. Aquellas personas que aprenden un idioma extranjero dentro de un aula de clases experimentan algunos problemas con el idioma cuando se enfrentan a él en una situación real en un país donde esta lengua es la lengua oficial. El tema aquí se sitúa en la poca comprensión de lo que se escucha debido a la variedad de vocabulario y expresiones que no han sido enseñadas en clase. Para nosotros los hablantes no nativos del idioma inglés por ejemplo es muy difícil comprender las palabras o términos coloquiales en inglés cuando viajamos a Estados Unidos o Inglaterra. Este tema de las jergas es un problema al cual nos enfrentamos en diferentes partes del mundo con sus respectivas lenguas. No se enmarca únicamente a un ámbito local. En nuestro país, las jergas varían según la región y también existen algunos términos que podrían considerarse como desconocidos por personas de un mismo país. Las jergas como vemos claramente están presentes en nuestro accionar diario en diferentes partes de nuestro país y del mundo. Son parte del habla diaria y deben ser tomadas en cuenta para el buen entender y hablar cotidiano. Muchas veces adoptamos las nuevas jergas por estar a la moda y a la par con los nuevos tiempos. Sin embargo, hay algunas jergas que permanecen tal cual con el tiempo. En la calle solemos escuchar jergas nuevas para nosotros pero que ya han sido incluidas socialmente por algún grupo de personas, ya sean profesionales, estudiantes, o alguien perteneciente a algún grupo social diferente al nuestro. Quizás estas jergas son nuevas para nosotros y sin habernos dado cuenta ya están incluidas en el hablar diario y queremos creer que nuestra jerga aún se sigue utilizando y no está desapareciendo lentamente. Es por esta razón que planeamos hacer un análisis contrastivo y descriptivo de aquellas jergas que siguen vigentes en la actualidad y ver cuáles de ellas ya está en desuso. Este análisis lo hicimos tomando un grupo de jergas peruanas de trabajos anteriores y encuestaremos a diferentes estudiantes universitarios pertenecientes a la Universidad César Vallejo y que estén cursando el primer año de estudios de la Escuela de Idiomas para saber si estas jergas aún están en uso en la actualidad y si están siendo usadas con el mismo significado de antes o este significado ha variado con el tiempo. El análisis se hizo durante el semestre 2019-1 y se tomaron una muestra de 100 jergas. Reina (2008), en su trabajo de investigación "Configuración de la jerga de los estudiantes de la licenciatura en lenguas modernas de la Pontificia Universidad Javeriana, desde una perspectiva sociolingüística", a fin de obtener su título de Licenciada en Lenguas Modernas, manifiesta los resultados obtenidos con respecto a la variable género, concluyendo que son las mujeres de la Licenciatura quienes utilizan con mayor frecuencia la jerga estudiantil. Según la muestra obtenida determina que los estudiantes de la Licenciatura utilizan préstamos lingüísticos para configurar su jerga estudiantil. Sin embargo, existe mayor uso de préstamos tomados más del inglés que del francés. Jaramillo (2013), en su investigación "Aspectos sociolingüísticos de la jerga del ejército de Colombia", en la Universidad Tecnológica 14 de Pereira, a fin de obtener su título de Licenciado en Español y Literatura, asevera entre una de sus conclusiones que cuanto más crezca la población de hablantes de las diversas lenguas, estas a su vez sufren cambios de transformación más difíciles y/o sistematizados, es obvio que dichos cambios no han sido fijados por variantes como el prestigio o la imposición lingüística; las variaciones se dan de forma natural y apartada a una observación progresiva del fenómeno. Según la hipótesis planteada seguida de las evidencias expuestas, se puede concluir diciendo que: "La forma de hablar de la comunidad del Ejército colombiano constituye un gremio que se une en relación a una jerga particular y transcrita". Gáfaro (1993) en su tesis titulado "Jerga Javeriana" pretende responder a la interrogante si realmente hay una jerga inherente a los estudiantes de Bogotá de la Pontificia Universidad Javeriana (1993). Esta investigación demostró que, la Universidad Javeriana es un campo bueno para la formación de palabras que la distinguen no 
sólo como espacio social específico sino como espacio educativo. El instrumento utilizado para dicho estudio logró ser muy viable, funcional y rápido, ya que se comprobó respuestas acertadas y reales a lo que se preguntaba. Asimismo, dicho trabajo de investigación brinda un glosario de base para investigaciones posteriores. El estudio de la jerga de los estudiantes de la Universidad César Vallejo de Trujillo se fundamenta en un corpus teórico que se desarrolla en la investigación a partir del análisis contrastivo de las variables; por tal motivo es importante tomar en cuenta ciertos principios sociolingüísticos que sirvieron en el análisis de las jergas peruanas como son:

El lenguaje, Maturana (1998) considera al lenguaje como fenómeno de la vida la cual pertenece al proceso de desarrollo evolutivo de la humanidad, refiriéndose de este modo a la relación entre lenguaje y el comportamiento humano. El autor usa el término lenguajear para indicar la relación entre ambas. Es decir, la relación entre lo que queremos comunicar a otros y con otros. Según Vicuña (1910) afirma que la lengua solamente sufre cambios léxicos más no cambios gramaticales. Es decir que con el tiempo y a pesar de los cambios léxicos que se den, las estructuras gramaticales se mantienen iguales. En base a la información previamente citada, podemos concluir que la lengua está sujeta a cambios causados por factores tanto internos inherentes a la propia lengua y externos como el ambiente en que este se rodea. Dentro de las diferentes definiciones que existen del término jerga, hemos considerado las que se mencionan a continuación tomando la definición de Bailey como la más acertada para nuestro tema de investigación. Por otro lado, Sanmartín (1998) define a la jerga como un lenguaje sectorial, relacionado con la creación de nuevas palabras ligadas a las diferentes profesiones, ocupaciones, etc. que realizan las personas. Esta afirmación que Sanmartín establece nos permite concluir que existen términos usados por un grupo específico de individuos quienes están constantemente creando nuevas palabras o términos en relación a dichas ocupaciones y/o actividades. Para poder entender de una mejor manera la terminología relacionada a nuestro objeto de investigación hemos tomado algunas diferencias entre el término jerga y otros más para su mejor entendimiento y cuyo detalle aparece a continuación. Con el término Jerga y Argot existen diferentes definiciones para esta palabra. Algunos autores incluso señalan que jerga y argot significan lo mismo. Para la Real Academia Española (2014), jerga y argot se usan para describir lo mismo. Sin embargo, para otros autores señalan diferencias entre ambas. Tales diferencias las podemos encontrar en diferentes autores como: Rodríguez (1981), quien define a jerga o argot como un lenguaje particular compartido por individuos que pertenecen al mismo grupo social o profesional. Después de haber citado a diferentes autores y haber analizado las diferencias entre argot y jerga podemos concluir que para esta investigación nos enfocaremos en el uso de las jergas de los estudiantes. Se consideró mencionar los propósitos de la jerga, según Partridge (1935) nos da una lista de 15 posibles razones para usar jerga como: Solo por diversión, por emulación, para ser diferente o novedoso, para evitar negatividad, para ser deslumbrante o sorprendente, para ser breve y conciso, para enriquecer el lenguaje (pasa entre los más educados), dar un aire de solidez, concreción al resumen, dar un punto adicional a una negativa o un rechazo, para reducir la excesiva seriedad de una conversación, para suavizar la tragedia, para estar a nivel coloquial, para facilitar las relaciones sociales, para mostrar que uno pertenece a una determinada escuela, profesión u oficio, ara que el mensaje no sea entendido por quienes lo rodean, estudiantes, criminales, políticos, etc. La definición de la jerga peruana se alinea a los mismos principios sociolingüísticos del lenguaje como se puede entender en base a toda la teoría recopilada en esta investigación. Hevia (2014) escribió varios libros sobre la jerga peruana y presenta dos puntos de vista: Una de ellas entiende que se trata de un código cerrado, considera a la jerga refractaria a la observación y, por añadidura, relega su práctica a grupos marginales o elitistas. La otra la imagina como un gran desborde, solo sabe vincularla con diversificaciones y excesos retóricos, subraya su acusado barroquismo y la imbarajable propagación de sus efectos. En resumen, para poder analizar y/o contrastar el uso de la jerga por los estudiantes universitarios, hay que definir los términos que están relacionados con este lenguaje coloquial. Será importante introducir las definiciones de las jergas peruanas más usadas para el propósito de esta investigación.

Es importante considerar la Jerga o peruanismo, Ángeles (2003) clasifica los peruanismos según a lo que se refieren o designan. Son voces que se refieren a la organización social, aluden a costumbres o actividades, designan animales, plantas, alimentos, objetos, bebidas o voces diversas. El lenguaje cotidiano muchas veces pasa desapercibido y los cambios que el idioma sufre son imperceptibles en la línea del tiempo. Para los involucrados con el aprendizaje de los idiomas y sobre todo para aquellos interesados en la 
lingüística es de suma importancia saber cómo el idioma se va transformando a través del tiempo. Las razones pueden ser motivo para investigaciones futuras; sin embargo, lo que sucede con las expresiones coloquiales de nuestra lengua materna (jergas) nos dan una visión más profunda de los cambios o mutaciones que han tenido a través del tiempo. El idioma es el alma de una cultura y como tal debe ser tomado en cuenta con la relevancia correspondiente que se merece. El español en particular es un idioma que últimamente se ha visto influenciado por múltiples factores, y es menester de los especialistas en los idiomas investigar dichos cambios, es por esta razón que esta investigación se justifica de manera teórica y metodológica pues realzamos la importancia del español en nuestra vida diaria y la información nos sirve para teorizar a profundidad en el idioma español y sus variantes. Nos hicimos la siguiente interrogante: ¿Cómo han variado las jergas peruanas de hace una década con las actuales? Como objetivo general nos planteamos el analizar si las jergas peruanas de hace una década han variado con el paso de los años comparándolas con las actuales. Dentro de los objetivos específicos que hemos considerado fueron: Identificar las jergas peruanas de uso coloquial de hace una década, asimismo identificar las jergas que siguen en vigencia en la actualidad y aquellas que ya no siguen en vigencia; y finalmente identificar posibles jergas o palabras equivalentes para aquellas jergas en desuso.

\section{MATERIAL Y MÉTODOS}

\subsection{Tipo y diseño de investigación}

Es una investigación de tipo cualitativa y de diseño descriptivo.

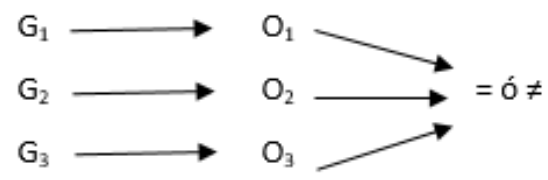

\subsection{Escenario de estudio}

Escuela de Idiomas - Universidad Cesar Vallejo - 2019

\subsection{Caracterización de participantes}

Los participantes de la presente investigación son 60 estudiantes del 1er ciclo de la carrera de Traducción e Interpretación

\subsection{Plan de análisis o trayectoria metodológica}

- Se creó un instrumento (Cuestionario).

- Se validó el cuestionario por 03 expertos.

- Se aplicó el instrumento a los participantes.

- Se recogió la información de dichos cuestionarios.

- Se tabuló la información obtenida y se procesó a través de Excel.

\subsection{Técnicas e instrumentos de recolección de información}

Técnica: Encuesta

Instrumento: Cuestionario

\subsection{Tratamiento de la información:} Unidades temáticas, categorización

\section{Unidades temáticas:}

- Listado de Jergas en uso, 2009.

- Listado de jergas en uso en la actualidad, 2019.

- Listado de palabras equivalentes a las jergas en desuso en la actualidad, 2019.

- Se realizó un Muestreo No Probabilístico Muestreo por conveniencia

\section{Categorización:}

Hevia (2014) define las jergas desde dos perspectivas, la primera que habla sobre un código cerrado y la otra la vincula con diversificaciones y excesos retóricos.

Las jergas en estudio forman parte de un estudio previo y se enmarcan dentro del lenguaje coloquial juvenil del año 2009.

\subsection{Mapeamiento}

El escenario donde este trabajó se realizó comprende a jóvenes estudiantes universitarios del 1er año de la carrera de Traducción que están en frecuente uso del lenguaje coloquial de acuerdo a su edad. La edad de mayoría de ellos fluctúa entre los 16 y 18 años. En la actualidad, con el avance de la tecnología y las redes sociales, el lenguaje cotidiano y coloquial también ha tomado nuevos rumbos en estos últimos años.

\subsection{Rigor científico}

A través de la historia se ha teorizado mucho sobre el lenguaje, su adqusición y desarrollo a través del tiempo. En tal sentido, Maturana (1998) considera al lenguaje como fenómeno de la vida la cual pertenece al proceso de desarrollo evolutivo de la humanidad, refiriéndose de este modo a la 
relación entre lenguaje y el comportamiento humano. Es bien sabido entonces que el lenguaje como parte de la cultura sufre cambios con el tiempo. Por consiguiente, se buscó la coherencia entre la teoría y la práctica con datos certeros, auditables y aplicables con fines netamente informativos y en pro al conocimiento más profundo del lenguaje.

\section{RESULTADOS}

Con respecto al primer objetivo y para efectos de la investigación se tomó en cuenta el siguiente listado de jergas peruanas usadas hace más de 10 años. Para efectos de esta investigación, se utilizó una lista de 70 jergas. Esta lista fue extraída de un trabajo disponible en la página web: https://blogs.deperu.com/chistes/significado-de-las-jergas-peruanas/

Tabla 1. Listado de las Jergas más comunes, 2009

\begin{tabular}{|c|c|}
\hline Jerga & Definición \\
\hline 1. achorado & persona de carácter desafiante. \\
\hline 2. afanar & enamorar. \\
\hline 3. aqarre & un beso casual \\
\hline 4. agarrado & fuerte, musculoso \\
\hline 5. aguja & Misio \\
\hline 6. algodón & Estar en algo \\
\hline 7. alucina & imagina \\
\hline 8. asado & enojado, molesto. \\
\hline 9. bacán & maravilloso. \\
\hline 10. bamba & falsificado \\
\hline 11. batería & Grupo de amigos cercanos. \\
\hline 12. cana & Cárcel \\
\hline 13. cabeceador & persona que no devuelve lo prestado. \\
\hline 14. cachaciento & persona que se mofa para fastidiar. \\
\hline 15. cachuelo & Trabajo temporal. \\
\hline 16. calabacita & chica tonta \\
\hline 17. caleta & Algo poco conocido. \\
\hline 18. causa & amigo cercano. \\
\hline 19. costilla & enamorada \\
\hline 20. chamba & Trabajo \\
\hline 21. chancón & persona que estudia mucho. \\
\hline 22. chancha & colecta \\
\hline 23. chape & Beso \\
\hline 24. chela & cerveza \\
\hline 25. chongo & diversión, prostíbulo. \\
\hline 26. choro & Ladrón \\
\hline 27. chupar & Beber \\
\hline 28. churro & hombre guapo \\
\hline 29. cutra & Dinero obtenido de forma ilícita. \\
\hline 30. chucki & Niño malcriado. \\
\hline 31. enyucar & Engañar, estafar. \\
\hline 32. fintero & que aparenta algo que no es. \\
\hline 33. florear & adular, alabar \\
\hline 34. gil & Tonto \\
\hline 35. huachafo & persona de malos gustos \\
\hline 36. huevo & Fácil \\
\hline 37. jatear & dormir \\
\hline 38. jerma & enamorada \\
\hline 39. jugadora & mujer promiscua \\
\hline 40. lechero & suertudo \\
\hline 41. lenteja & Lento \\
\hline 42. lorear & Hablar \\
\hline 43. luca & moneda \\
\hline 44. Ilegar & no importar \\
\hline 45. maldito & muy bueno \\
\hline 46. mancha & multitud \\
\hline 47. mariachi & marido \\
\hline 48. manyar & entender \\
\hline 49. mecharse & Pelearse con alguien. \\
\hline
\end{tabular}




\begin{tabular}{cc}
\hline 50. misio & sin dinero \\
\hline 51. monse & Tonto \\
\hline 52. mosca & Alerta \\
\hline 53. papaya & fácil \\
\hline 54. paia & Bonito \\
\hline 55. palta & vergüenza \\
\hline 56. pata & Amigo \\
\hline 57. pavo & Tonto \\
\hline 58. pendeio & persona astuta \\
\hline 59. piña & Que tiene mala suerte. \\
\hline 60. pituco & persona adinerada \\
\hline 61. quina & cincuenta céntimos \\
\hline 62. rayarse & volverse loco \\
\hline 63. resaca & malestar físico después de una \\
\hline 64. roche & borrachera \\
\hline 65. sapo & vergüenza \\
\hline 66. taba & individuo listo \\
\hline 67. tela & Zapato \\
\hline 68 tombo & sin dinero \\
\hline 69. tono & agente de policía \\
\hline 70. tranca & Fiesta \\
\hline
\end{tabular}

Nota. Las palabras de la lista son una muestra extraída del trabajo de investigación titulado iHabla, jugador! Gajes y oficios de la jerga peruana. perteneciente a Julio Hevia (2014).

Para la palabra ACHORADO, de los 60 alumnos encuestados 36 alumnos aún siguen usando esta jerga hasta la actualidad.

Para la palabra AFANAR, de los 60 alumnos encuestados 42 alumnos aún siguen usando esta jerga hasta la actualidad.

Para la palabra AGARRE, de los 60 alumnos encuestados 43 alumnos aún siguen usando esta jerga hasta la actualidad

Para la palabra ALUCINA, de los 60 alumnos encuestados 42 alumnos aún siguen usando esta jerga hasta la actualidad.

Para la palabra AGUJA, de los 60 alumnos encuestados 37 alumnos aún siguen usando esta jerga hasta la actualidad.

Para la palabra ASADO, de los 60 alumnos encuestados 47 alumnos aún siguen usando esta jerga hasta la actualidad.

Para la palabra BACÁN, de los 60 alumnos encuestados 47 alumnos aún siguen usando esta jerga hasta la actualidad.

Para la palabra BAMBA, de los 60 alumnos encuestados 37 alumnos aún siguen usando esta jerga hasta la actualidad.

Para la palabra CHUPAR, de los 60 alumnos encuestados 36 alumnos aún siguen usando esta jerga hasta la actualidad.

Para la palabra CHELA, de los 60 alumnos encuestados 36 alumnos aún siguen usando esta jerga hasta la actualidad.

Para la palabra CALETA, de los 60 alumnos encuestados 41 alumnos aún siguen usando esta jerga hasta la actualidad.

Para la palabra CAUSA, de los 60 alumnos encuestados 35 alumnos aún siguen usando esta jerga hasta la actualidad

Para la palabra CUTRA, de los 60 alumnos encuestados 34 alumnos aún siguen usando esta jerga hasta la actualidad.

Para la palabra CHAMBA, de los 60 alumnos encuestados 38 alumnos aún siguen usando esta jerga hasta la actualidad

Para la palabra CHANCHA, de los 60 alumnos encuestados 44 alumnos aún siguen usando esta jerga hasta la actualidad.

Para la palabra CHANCÓN, de los 60 alumnos encuestados 50 alumnos aún siguen usando esta jerga hasta la actualidad.

Para la palabra CHAPE, de los 60 alumnos encuestados 49 alumnos aún siguen usando esta jerga hasta la actualidad.

Para la palabra CHELA, de los 60 alumnos encuestados 43 alumnos aún siguen usando esta jerga hasta la actualidad

Para la palabra CHONGO, de los 60 alumnos encuestados 33 alumnos aún siguen usando esta jerga hasta la actualidad.

Para la palabra CHORO, de los 60 alumnos encuestados 48 alumnos aún siguen usando esta jerga hasta la actualidad.

Para la palabra CHUPAR, de los 60 alumnos encuestados 36 alumnos aún siguen usando esta 
jerga hasta la actualidad.

Para la palabra CHUCKI, de los 60 alumnos encuestados 50 alumnos aún siguen usando esta jerga hasta la actualidad.

Para la palabra CHURRO, de los 60 alumnos encuestados 43 alumnos aún siguen usando esta jerga hasta la actualidad.

Para la palabra GIL, de los 60 alumnos encuestados 41 alumnos aún siguen usando esta jerga hasta la actualidad.

Para la palabra FINTERO, de los 60 alumnos encuestados 38 alumnos aún siguen usando esta jerga hasta la actualidad.

Para la palabra FLOREAR, de los 60 alumnos encuestados 53 alumnos aún siguen usando esta jerga hasta la actualidad.

Para la palabra HUACHAFO, de los 60 alumnos encuestados 40 alumnos aún siguen usando esta jerga hasta la actualidad.

Para la palabra JATEAR, de los 60 alumnos encuestados 36 alumnos aún siguen usando esta jerga hasta la actualidad.

Para la palabra LENTEJA, de los 60 alumnos encuestados 34 alumnos aún siguen usando esta jerga hasta la actualidad.

Para la palabra LUCA, de los 60 alumnos encuestados 37 alumnos aún siguen usando esta jerga hasta la actualidad.

Para la palabra LLEGAR, de los 60 alumnos encuestados 47 alumnos aún siguen usando esta jerga hasta la actualidad.

Para la palabra MANYAR, de los 60 alumnos encuestados 37 alumnos aún siguen usando esta jerga hasta la actualidad.

Para la palabra MANCHAR, de los 60 alumnos encuestados 43 alumnos aún siguen usando esta jerga hasta la actualidad.

Para la palabra MECHARSE, de los 60 alumnos encuestados 43 alumnos aún siguen usando esta jerga hasta la actualidad.

Para la palabra MARIACHI, de los 60 alumnos encuestados 35 alumnos aún siguen usando esta jerga hasta la actualidad.

Para la palabra MISIO, de los 60 alumnos encuestados 54 alumnos aún siguen usando esta jerga hasta la actualidad.

Para la palabra MONSE, de los 60 alumnos encuestados 37 alumnos aún siguen usando esta jerga hasta la actualidad.

Para la palabra MOSCA, de los 60 alumnos encuestados 37 alumnos aún siguen usando esta jerga hasta la actualidad.

Para la palabra PALTA, de los 60 alumnos encuestados 53 alumnos aún siguen usando esta jerga hasta la actualidad.

Para la palabra PATA, de los 60 alumnos encuestados 37 alumnos aún siguen usando esta jerga hasta la actualidad.

Para la palabra PAPAYA, de los 60 alumnos encuestados 40 alumnos aún siguen usando esta jerga hasta la actualidad.

Para la palabra PENDEJ0, de los 60 alumnos encuestados 46 alumnos aún siguen usando esta jerga hasta la actualidad.

Para la palabra PIÑA, de los 60 alumnos encuestados 56 alumnos aún siguen usando esta jerga hasta la actualidad

Para la palabra PITUCO, de los 60 alumnos encuestados 50 alumnos aún siguen usando esta jerga hasta la actualidad.

Para la palabra QUINA, de los 60 alumnos encuestados 39 alumnos aún siguen usando esta jerga hasta la actualidad.

Para la palabra RESACA, de los 60 alumnos encuestados 51 alumnos aún siguen usando esta jerga hasta la actualidad.

Para la palabra ROCHE, de los 60 alumnos encuestados 50 alumnos aún siguen usando esta jerga hasta la actualidad.

Para la palabra SAPO, de los 60 alumnos encuestados 33 alumnos aún siguen usando esta jerga hasta la actualidad.

Para la palabra TOMBO, de los 60 alumnos encuestados 34 alumnos aún siguen usando esta jerga hasta la actualidad.

Para la palabra TONO, de los 60 alumnos encuestados 43 alumnos aún siguen usando esta jerga hasta la actualidad.

Con respecto al tercer y cuarto objetivo específico que fue identificar las jergas peruanas que no siguen en vigencia y sus equivalentes en la actualidad, se aplicó el instrumento (Anexo 01) a 60 alumnos del primer ciclo de Traducción e Interpretación de la Universidad César Vallejo, obteniendo los siguientes resultados:

En la palabra AGARRADO cuyo significado hace 10 años fue fuerte, musculoso, actualmente está en desuso; sin embargo se encontraron los siguientes términos equivalentes para dicha palabra: "cuadrado, cuerpón, maceta, fortachón, chapado, mamado y musculoso."

En la palabra ALGODóN cuyo significado hace 10 
años fue estar en algo, actualmente está en desuso; sin embargo se encontraron los siguientes términos equivalentes para dicha palabra: "amigos con derecho, positivo, a nada, en planes y se defiende"

En la palabra BATERIA cuyo significado hace 10 años fue grupo de amigos cercanos, actualmente está en desuso; sin embargo se encontraron los siguientes términos equivalentes para dicha palabra: "causa, mi hermano, gente, brother, banda, bros, people, pata y amix".

En la palabra CABECEADOR cuyo significado hace 10 años fue persona que no devuelve lo prestado, actualmente está en desuso; sin embargo se encontraron los siguientes términos equivalentes para dicha palabra: "vivo, choro, tramposo, conchudo, moroso, sin vergüenza, ratero, estafador, pendejo, atorrante y falla".

En la palabra CALABACITA cuyo significado hace 10 años fue chica tonta, actualmente está en desuso; sin embargo se encontraron los siguientes términos equivalentes para dicha palabra: "boba, tonta, nerd, mongolita, ilusa, estúpida, bruta, sonsa".

En la palabra CANA cuyo significado hace 10 años fue: ir a prisión, actualmente está en desuso; sin embargo se encontraron los siguientes términos equivalentes para dicha palabra: "canadá, prisión".

En la palabra CACHACIENTO cuyo significado hace 10 años fue: persona que se mofa de los demás, actualmente está en desuso; sin embargo se encontraron los siguientes términos equivalentes para dicha palabra: "jodido, espanto, candelero, picón, chacotero, ardiloso, burlón".

En la palabra CACHUELO cuyo significado hace 10 años fue: trabajo temporal , actualmente está en desuso; sin embargo se encontraron los siguientes términos equivalentes para dicha palabra: "part time y chambita".

En la palabra CostILLA cuyo significado hace 10 años fue: enamorada, actualmente está en desuso; sin embargo se encontraron los siguientes términos equivalentes para dicha palabra: "flaca, enamorada y jerma".

En la palabra ENYUCAR cuyo significado hace 10 años fue: engañar y estafar, actualmente está en desuso; sin embargo se encontraron los siguientes términos equivalentes para dicha palabra: "engañar, timar y estafar".
En la palabra HUEVo cuyo significado hace 10 años fue: fácil, actualmente está en desuso; sin embargo se encontraron los siguientes términos equivalentes para dicha palabra: "papayita, mantequilla, easy y lata".

En la palabra JERMA cuyo significado hace 10 años fue: enamorada, actualmente está en desuso; sin embargo se encontraron los siguientes términos equivalentes para dicha palabra: "flaca y enamorada".

En la palabra JUGADORA cuyo significado hace 10 años fue: mujer promiscua, actualmente está en desuso; sin embargo se encontraron los siguientes términos equivalentes para dicha palabra: "pendeja, facilita, positiva y prosti".

En la palabra LECHERO cuyo significado hace 10 años fue: suertudo, actualmente está en desuso; sin embargo se encontraron los siguientes términos equivalentes para dicha palabra: "bendito".

En la palabra LOREAR cuyo significado hace 10 años fue: hablar, actualmente está en desuso; sin embargo se encontraron los siguientes términos equivalentes para dicha palabra: "florear, meter floro, ser un conversalón y cotorrear".

En la palabra MALDITO cuyo significado hace 10 años fue: muy bueno, actualmente está en desuso; sin embargo se encontraron los siguientes términos equivalentes para dicha palabra: "chevere, bacán, buena suerte, buenazo, crack y pro".

En la palabra PAJA cuyo significado hace 10 años fue: bonito, actualmente está en desuso; sin embargo se encontraron los siguientes términos equivalentes para dicha palabra: "bacán, piola, nice y churro".

En la palabra PAVO cuyo significado hace 10 años fue: tonto, actualmente está en desuso; sin embargo se encontraron los siguientes términos equivalentes para dicha palabra: "gil, idiota, y bestia".

En la palabra RAYARSE cuyo significado hace 10 años fue: volverse loco, actualmente está en desuso; sin embargo se encontraron los siguientes términos equivalentes para dicha palabra: "flipar y le agarró".

En la palabra TABA cuyo significado hace 10 años fue: zapato, actualmente está en desuso y no tiene equivalente.

En la palabra TELA cuyo significado hhace 10 años fue: sin dinero, actualmente está en desuso; sin embargo se encontraron los siguientes términos 
equivalentes para dicha palabra: "delgado, estar en nada, aguja, misión imposible y Chihuan".

En la palabra TRANCA cuyo significado hace 10 años fue: borrachera, actualmente está en desuso; sin embargo, se encontraron los siguientes términos equivalentes para dicha palabra: "resaca".

Se ha podido notar que los estudiantes, al momento de colocar algún equivalente para las jergas en desuso, utilizan algunas palabras que provienen de las redes sociales, especialmente de algunos países de habla hispana como Méjico - España. No se han usado préstamos lingüísticos en otros idiomas diferentes al español como sí sucedió en el trabajo de Reina (2018) titulado Configuración de la jerga de los estudiantes de la licenciatura en lenguas modernas de la Pontificia Universidad Javeriana, desde una perspectiva sociolingüística.

Asimismo, los resultados de la investigación demuestran que, a pesar de los años transcurridos, existe un grupo de jergas de la muestra que sigue siendo usado por los jóvenes. Esto reafirma lo dicho por Jaramillo (2013) que los grupos sociales determinados se unen en relación a una jerga en particular.

El cuestionario en algunos casos parecía ser muy extenso para unos pocos estudiantes y se puede percibir en la manera que contestaron y completaron la información. Esto concuerda con el trabajo hecho por Gáfaro (1993) donde también contestaron de una manera poco responsable. Sin embargo, reafirma el uso común de las jergas dentro de la vida universitaria.

\section{CONCLUSIÓN}

Con el paso de los años, 22 jergas de un total de 70 han variado o están siendo substituidas por nuevos términos equivalentes. Este hallazgo nos muestra que, del total de estudiantes encuestados, más de la mitad de ellos han dejado de usar estos 22 términos a la actualidad.

El listado de jergas utilizadas en esta investigación para la comparación y análisis fueron tomados de manera aleatoria en un número de 70 de una investigación realizada hace 10 años.

Las jergas que siguen en vigencia y aún están en uso son 48 del total de 70 , lo que significa que más del $50 \%$ de ellas permanece con el significado intacto comparándolas en el tiempo.

Para aquellas jergas en desuso, se muestra algunos términos equivalentes sugeridos por los participantes de la investigación.

\section{REFERENCIAS BIBLIOGRÁFICAS}

Ángeles, C. (2003). Los peruanismos en la literatura peruana. Lima: Universidad Inca Garcilaso de la Vega, Fondo editorial.

Bailey, R. (1985). South African English Slang: Form, functions, and origins. South African Journal of Linguistics.

Bendezú, G. (1975). Vocabulario hampesco. Perú: Edit. Kuntur.

Bendezú, G. (1977). Argot limeño o jerga criolla del Perú. Lima: Librería importadora, editora y distribuidora Lima.

Eckert, P. (2000) Linguistic Variation: Social Practice. New York: Blackwell Publishers.

Fasold, R. (1990) Sociolinguistics of Language. Oxford: Basil Blackwell.

Gáfaro, A. (1993). Jerga Javeriana (tesis de licenciatura). Pontificia Universidad Javeriana de Bogotá, Colombia.

García, J. (1994). Lenguajes marginales. Análisis y vocabularios.
García, E. (2007). Reflexiones y propuestas para la ciencia política. Editorial universitaria UNAM.

Hevia, J. (2014). iHabla, jugador! Gajes y oficios de la jerga peruana. Lima: Taurus.

Jaramillo, J. (2013). Aspectos sociolingüísticos de la jerga del Ejército de Colombia (tesis de Licenciatura en español y Literatura). Universidad Tecnológica de Pereira, Colombia.

Maturana, H. (1998). Emociones y lenguaje en educación y política. Bogotá, Tercer Mundo.

Menéndez, G. (1990). Dialectología y sociolingüística española. España: Edit. Universidad de Alicante.

Milroy, J. (1997). Varieties and Variation. In F. Coulmas (ed.). A Handbook of Sociolinguistics. Oxford: Blackwell.

Montes, J. (1995). Dialectología general e hispanoamericana. Bogotá: Edit. Caro y cuervo. Partridge, E. (1935) Slang Today and Yesterday. London: 
Routledge and Kegan Paul Ltd.

Ramírez, L. (1996). Estructura y funcionamiento del lenguaje. Lima, Derrama Magisterial.

Ramírez, N. (2008). Jerga Estudiantil Universitaria (tesis de licenciatura). Universidad de Costa Rica, Costa Rica.
Schvarstein, L. (2003). Inteligencia Social De Las Organizaciones. Desarrollando Las Competencias Necesarias Para EI Ejercicio Efectivo De La Responsabilidad Social. Buenos Aires: Paidós.

Vicuña, J. (1910). COA: Jerga de los delincuentes chilenos. Santiago de Chile: Edit. Universitaria Bandera.

Recibido: 18 marzo 2019 | Aceptado: 20 mayo 2019 